Ökobilanzierung aus Sicht der Verbraucherverbände

\title{
Erwartungen nicht erfüllt
}

\begin{abstract}
Von einer Ökobilanz wird eine Antwort erhofft auf die Frage, wie sich wirtschaffliche Aktivitäten des Menschen auf die Umwelt auswirken. Dabei soll der erwartete Erkenntnisgewinn mit dem Ziel genutzł werden, ökologische Lasten zu verringern. Ein besonderes Interesse hat die kritische Öffentlichkeit in den rund 20 Jahren der Ökobilanzdiskussion dabei den vergleichenden Produkt-Ökobilanzen entgegengebracht.
\end{abstract}

$\mathrm{D}$

Von Bodo Tegethoff ie Verbraucherverbände versprachen sich seinerzeit von der vergleichenden Produkt-Ökobilanz eine naturwissenschaftlich abgesicherte Basis für die Produktberatung, aber auch eine weitestgehend objektivierte Grundlage für die Umweltpolitik, mithin eine Art Kompaß auf dem schwierigen Weg in Richtung einer nachhaltigen Entwicklung bzw. eines nachhaltigen Konsums. Allein, diese hohen Erwartungen konnten bisher jedenfalls nicht erfüllt werden. Gründe dafür gibt es viele. Nicht standardisierte Bilanzierungsvorschriften „nach Art des Hauses" müssen zwangsläufig zu subjektiven Ergebnissen führen. Vor allem von Herstellern bzw. von Branchen durchgefiihrte Bilanzen ließen recht regelmäßig die eigenen Produkte in besserem Licht erscheinen als die der Konkurrenz. Neben klaren wirtschaftlichen Interessen gab es zusätzlich ein mehr oder minder wissenschaftlicher Disput um die beste Methodik. Und schließlich waren - und sind - Wirkungen der Schadstoffe auf die Ökosysteme vielfach nicht bekannt. Eine Antwort in mathematischer Exaktheit kann eine Ökobilanz daher nicht geben. Anders ausgedrückt, aus Sicht der Verbraucherverbände lag eine der Ursachen der teilweise enttäuschten Erwartungen im Zusammenhang mit Ökobilanzen in der Vergangenheit darin, daß zu einseitig auf die Naturwissenschaften vertraut wurde. Naturwissenschaftler können uns sagen, wie sich FCKWs in der Stratosphäre auswirken oder Phosphat in der Nordsee. Ihre Stärke, erst recht ihre Aufgabe, kann aber nicht darin bestehen, den Streit $\mathrm{zu}$ entscheiden, welche Beeinträchtigungen der Umwelt und der Gesundheit die Gesellschaft hinzunehmen hat. Ökologen können uns keine Antwort darauf geben, was „besser“ ist, die Ausdehnung des
Ozonlochs oder die Überdüngung der Nordsee. Antworten auf diese Fragen lassen sich nur im Konsens der gesellschaftlichen Gruppen, allerdings auf der Basis einer wissenschaftlichen Datenlage, finden.

Unter der Voraussetzung, daß für die Ökobilanzierung ein verbindlicher Standard festgelegt wird, unter dem die verschiedenen Phasen wie Festlegung des Ziels und Untersuchungsrahmens, Sachbilanz, Wirkungsabschätzung und Auswertung, ablaufen, werden seriös durchgefuihrte Ökobilanzen durchaus belastbare Ergebnisse liefern können, die für die unternehmensinterne Produktoptimierung, die Verbraucherberatung in Umweltfragen, aber auch für die Weiterentwicklung der Umweltpolitik brauchbar sind.

Für die Verbraucherverbände ist die „Auswertung" als der letzte Schritt einer Ökobilanz eine wichtige Schnittstelle zwischen den zuvor ermittelten naturwissenschaftlichen Fakten, technischen Erfordernissen und verbindlichen Konventionen einerseits und den Handlungsempfehlungen an die Akteure andererseits. Dem Auswertungsschritt kommt damit eine besondere Bedeutung im Rahmen der gesellschaftlichen Kommunikation über Umweltprobleme zu. Dabei ist uns klar, daß die Bewertungen wegen der ihnen notwendigerweise innewohnenden Subjektivität nicht standardisierbar sind. Gleichwohl muß das Verfahren standardisiert werden, nach dem die Bewertung stattfinden soll. Jede Ökobilanz, die für sich beansprucht, Konsum- und/oder Handlungs- empfehlungen für Verbraucher herzuleiten, muß auch die Möglichkeit beinhalten, die Verbraucherseite zumindest in den Prozeß der Bewertung einzubeziehen. Durch sogenannte Projektbeiräte, die auch anderen gesellschaftlichen Gruppen offenstehen sollten, läßt sich dies in der Praxis umsetzen.
Gibt es Abhilfe?

Wenn die Verbraucherverbände sich dennoch nicht enttäuscht von der Ökobilanzierung abwenden, dann deswegen, weil bessere Alternativen nicht in Sicht sind. Was die Standardisierung von Ökobilanzen anbelangt, sind aus unserer Sicht deutliche Fortschritte erkennbar. Auf der Ebene der internationalen Normung (ISO) ist dieser Prozeß mit der Normenreihe 14040 ff auf gutem Weg.

Ebenso wie die Herstellung, Nutzung und Entsorgung von Produkten bewegen sich auch Ökobilanzen nicht im luftleeren Raum. Ein hoher Wasserverbrauch in einer semi-ariden Region beispielsweise ist ökologisch schwerwiegender als in einer feuchtgemäßigten Zone der Erde. Auch spricht vieles dafür, zwischen reversiblen und irreversiblen Umweltschädigungen $\mathrm{zu}$ unterscheiden sowie zwischen lokalen, regionalen und globalen Wirkungen. Der „Treibhauseffekt" würde dann in einer Ökobilanz stärker zu Buche schlagen als eine lokale Beeinträchtigung durch Lärm. Hinsichtlich der Bewertungsmethodik unterstiitzen die Verbraucherverbände daher den Vorschlag des Umweltbundesamtes, die drei Kriterien ,ökologische Gefährdung“, „ökologische Knappheit“ und ,spezifischer Beitrag" zu wählen und in geeigneter Weise zu operationalisieren. Auch der Ansatz ,distance to target“ (Entfernung zum Umweltziel) oder frei übersetzt ,wie zukunftsfähig ist ein Produkt?" ist nicht nur richtig, sondern auch bereits bei einigen Wirkungskategorien, wie Treibhauseffekt und stratosphärischer Ozonabbau, hinreichend naturwissenschaftlich abzustïtzen. Was häufig fehlt, ist die Fixierung der Umweltziele (targets), ohne die es natürlich nicht möglich ist, eine Ent-

\begin{tabular}{|c|}
\hline Ökologische \\
Wirtschaftsforschung \\
im 21. Jabrbundert \\
ist das Thema des Informationsdienstes \\
Ökologisches Wirtschaften $1 / 1998$. \\
Wenn Sie potentielle Beiträge haben, \\
wenden Sie sich bitte an die Redaktion!
\end{tabular}


fernung (distance) anzugeben. Hier ist die Umweltpolitik unserer Auffassung nach in dringendem Zugzwang.

\section{Ökobilanzpolitik}

Nach unserer Überzeugung handelt es sich bei Aussagen, die auf der Grundlage geeigneter Ökobilanzen getroffen werden, nicht um die Diskriminierung von Produkten. Diskriminierung hieße nämlich, eine ungerechtfertigte Unterscheidung oder Ausgrenzung zu treffen. Sinn und Zweck einer seriösen Ökobilanz ist hingegen, ökologisch gerechtfertigte Differenzierungen vorzunehmen. Daher halten wir Ökobilanzen für durchaus geeignet, um Produkte zu beurteilen und gegebenenfalls auch umweltpolitische Maßnahmen einzuleiten.

Teile der Industrie scheinen hier anderer Meinung zu sein. Staatliches oder besser gesagt gesamtgesellschaftlich gewolltes Stoffstrommanagement oder eine entsprechende Produktpolitik werden abgelehnt. Aus dieser Motivation heraus werden Daten zurückgehalten, um Umweltpolitik auf der Grundlage von Ökobilanzen $\mathrm{zu}$ verhindern. Insbesondere manche im Verpackungssektor tätigen Unternehmen, vom Rohstofferzeuger über Verpackungshersteller bis zum Abfiiller, sekundiert von ihren Branchenverbänden, hüten ihre Betriebsgeheimnisse gut. Ziel dieser Strategie ist die ersatzlose Demontage der Mehrwegquote in der geltenden Verpackungsverordnung. Dabei müßte allen Beteiligten klar sein, daß „Einweg“ nicht immer schlecht, „Mehrweg“ nicht immer und für alle abzufüllenden Produkte gut ist.

Gerade eine gerechte Weichenstellung hängt von belastbaren Ökobilanzstudien ab. Wer hier Daten zurückhält, darf sich später nicht beklagen, wenn ,die Politik“ mangels Daten Rechtsnormen verabschiedet, mit denen bestimmte Verpackungssysteme diskriminiert werden.

\section{Der Autor}

Bodo M. Tegethoff ist Diplom-Agraringenieur und seit 1990 Umwelireferent bei der Arbeitsgemeinschaft der Verbraucherverbände, Die AgV ist der Dachverband der deutschen Verbraucherorganisationen. Die Arbeitsgebiete des Autors sind Abfall, Chemikalien im Haushalt, umweltrelevante Agrarfragen, nachhaltiger Konsum.

Kontakt: AgV, Heilsbachsir. 20, 53123 Bonn, Tel. 0228/6489-138, Fax 0228/644258.

Ökobilanzen und Stoffstrommanagement

\title{
Erhöhte ökologische Richtungssicherheit
}

\begin{abstract}
Die Ökobilanz wurde zu einem ausgefeilten Informationsinstrument weiterentwikkelt, doch im Hinblick auf die Einbettung in betriebliche Entscheidungsprozesse und die Umsetzung der Ergebnisse weist sie deutliche Defizite auf. Im Umweltmanagement gewinnt wiederum die ökologische Bewertung und Optimierung von Produkten an Bedeutung, ohne groß auf Ökobilanzen zurückzugreifen. Durch eine Verbindung von Ökobilanz und Stoffstrommanagement könnten sowohl in bezug auf das Umsetzungsdefizit der Ökobilanz als auch die ökologische Richtungssicherheit von Maßnahmen des Stoffstrommanagements Verbesserungen erzielt werden.
\end{abstract}

Von Kathrin Ankele n den letzten Jahren wurden Ökobilanzen methodisch erheblich weiterentwickelt (1). Dabei entstand jedoch ein zunehmend schwer nachvollziehbares Expertensystem, nicht nur für Außenstehende, sondern auch für die Mehrzahl der Mitarbeiter und Mitarbeiterinnen in den beteiligten Unternehmen. Wie die komplexen und komplizierten Informationen aus Ökobilanzen innerhalb von Unternehmen in Entscheidungsprozesse der Produktentwicklung und -optimierung einfließen können, blieb bislang weitgehend unbeachtet und außerhalb der Methodenentwicklung (2). Es überrascht daher nicht, daß die Rückkopplung zum Umweltmanagement unterentwickelt ist und konkrete Verbesserungsvorschläge, sofern sie in Ökobilanzen gemacht werden, im Vergleich $\mathrm{zu}$ den vorangestellten detaillierten Berechnungen eher banal erscheinen.

Das handlungsleitende und -gestaltende Potential der Ökobilanz wird auf diese Weise nicht voll ausgeschöpft. Sinnvoll wäre die Weiterentwicklung von einem statischen zu einem prozeßbegleitenden Informationsinstrument, wobei die Umsetzung von Erkenntnissen größere Bedeutung erlangt. Ob dazu jeweils Ökobilanzen in voller Länge durchgefuihrt werden müssen, kann an dieser Stelle nicht näher ausgefuihrt werden. Diskutiert werden in diesem Zusammenhang methodische Weiterentwicklungen wie „Streamlined LCA“ oder Screeningverfahren, die nach einer ersten Ökobilanz, die eine vollständige Datenbasis schafft, zum Einsatz kommen könnten (3).

Durch die Arbeiten der Enquete-Kommission „Schutz des Menschen und der Umwelt“ wurde das Konzept des Stoffstrommanagements ent- wickelt, das die „(...) zielorientierte, verantwortliche, ganzheitliche und effiziente Beeinflussung von Stoffströmen oder Stoffsystemen (beschreibt), wobei die Zielvorgaben aus dem ökologischen und ökonomischen Bereich kommen, unter Berücksichtigung der sozialen Aspekte. Die Ziele werden auf betrieblicher Ebene, in der Kette der an einem Stoffstrom beteiligten Akteure oder auf der staatlichen Ebene entwickelt" (4). Hier wurde also eine Methode entwickelt, die sich auf Stoff- oder Produktketten erstreckt und den Fokus auf das zwischen- und überbetriebliche Managen dieser Stoffströme legt.

Wirtschaftliche Entscheidungen werden i. d. R. unter Unsicherheit und unvollständiger Datenlage getroffen, da vollständige Informationen niemals vorliegen. Die Verfügbarkeit und Nutzung von ökologischen Informationen ist jedoch notwendig, um zielgerichtet ökologische Optimierungspotentiale identifizieren zu können und die Effektivität der getroffenen Maßnahmen im Hinblick auf die erzielten Umweltentlastungen beurteilen zu können. Die Analyse einer Vielzahl von Praxisbeispielen des Stoffstrommanagements (5) hat gezeigt, daß für den Erfolg der Stoffstrommanagementprojekte darïber hinaus die Aspekte Organisation und Motivation der beteiligten Akteure eine wesentliche Rolle spielen.

\section{Zusammenhang zwischen Ökobilanz und Stoffstrom- management}

Die These ist, daß die Effektivität von Ökobilanzen durch die systematische Einbettung in betriebliche Entscheidungsprozesse erhöht 
(c) 20I0 Authors; licensee IÖW and oekom verlag. This is an article distributed under the terms of the Creative Commons Attribution Non-Commercial No Derivates License (http://creativecommons.org/licenses/by-nc-nd/3.o/), which permits unrestricted use, distribution, and reproduction in any medium, provided the original work is properly cited. 